\title{
Receiver tube heat transfer analysis of a CCP collector designed based on branched fractals geometry
}

\author{
Angélica Palacios, Darío Amaya*, Olga Ramos \\ (GAV Research Group, Universidad Militar Nueva Granada, Bogota 110111, Colombia)
}

\begin{abstract}
For the development of systems related to renewable energy and specifically for thermo-solar energy, it is essential to study and analyze the different thermal phenomena of heat transfer, in search of the best conditions that allow strengthening the designs. Phenomena such as radiation, convection, and conduction are highly studied, from the field of materials to the field of infrastructure, finding day to day optimal solutions that guarantee a great use of the solar resource available on the earth's surface. This study presented the results of heat transfer analysis on a receiver pipe in a CCP; designed using the theory of branched fractal geometry to improve the heat transfer associated with concentrating solar capture systems. A fractal structure as a branch or arboreal fractal type network, influenced thermal and electrical parameters, as electrical resistance, thermal resistance, and convective heat transfer, so increasing the level branch heat transfer can be enhancement along of the pipe. The use of renewable energies in agriculture benefits the industrial processes that require a continuous power system, due to climatic conditions and geographical location is not a guarantee in food production fields, which reason solar capture systems contribute to food dehydration processes, water heating, refrigeration, and energy production.
\end{abstract}

Keywords: heat transfer, fractal geometry, solar collector, fractality, fractal dimension, parabolic cylindrical concentrator DOI: $10.25165 /$ j.ijabe.20201305.4733

Citation: Palacios A, Amaya D, Ramos O. Receiver tube heat transfer analysis of a CCP collector designed based on branched fractals geometry. Int J Agric \& Biol Eng, 2020; 13(5): 56-62.

\section{Introduction}

The human being has always considered nature and its phenomena as sources of inspiration for the development and problem solution in different knowledge areas ${ }^{[1]}$. One of the most attractive natural mechanisms for science and engineering is the heat and mass transfer, whereby different structures found in biological systems can be replicated and improved to make artificial systems much more efficient ${ }^{[2]}$.

Within the biological structures with large heat/mass transport properties $^{[3,4]}$, and great superiority in heat conduction are branched networks ${ }^{[5]}$. These geometries are typically found in trees, leaves, rays, neuronal dendrites, terrestrial fractures, and in the respiratory systems of mammalian animals ${ }^{[6]}$.

However, these geometries cannot be described by classical Euclidean laws, due to their highly irregular property with small arbitrary scales ${ }^{[7]}$. Another of the great properties that characterize fractal structures is self-similarity and the fractal dimension; the latter is usually adopted to quantitatively study the irregularities of fractal surfaces, its value has to vary from 2 to 3 depending on the roughness of the surface ${ }^{[8,9]}$.

Within the most known areas of knowledge with applications of fractal structures, are the economy and finance through the study of market behavior ${ }^{[10]}$; hydrodynamics, with porous fractal structures in fluids ${ }^{[11]}$, geoscience, in the study of the

Received date: 2018-10-24 Accepted date: 2020-07-02

Biographies: Angélica Palacios, PhD candidate, research interest: sustainable energy, renewable energies, solar collectors and fractal structures in nature, Email: u1801712@unimilitar.edu.co; Olga Ramos, Associate Professor, research interest: mechatronics, control and industrial automation, Email: olga.ramos@unimilitar.edu.co.

*Correspondingn author: Darío Amaya, Associate Professor, research interest: robotics, mechatronics and automation areas. GAV Research Group, Universidad Militar Nueva Granada, Bogota 110111, Colombia. Tel: +57-3204912106, Email: dario.amaya@unimilitar.edu.co. morphological surfaces of rocks or Earth ${ }^{[12,13]}$. In basic and applied sciences such as physics ${ }^{[14]}$ and medicine ${ }^{[15]}$; in turn, there are multiple developments in the field of engineering, related to materials ${ }^{[16]}$, image processing ${ }^{[17,18]}$, communications and signals ${ }^{[19]}$, the study of environmental conditions ${ }^{[20]}$, among others.

Some works have been developed concerning the present work subject, $\mathrm{Xu}$ et al. ${ }^{[21,22]}$ developed the modeling of heat transport properties in branched fractal type networks based on the laws of scale in electrical conductivity, heat conduction and heat transfer by convection for both laminar and turbulent flow.

Similarly, Luo et al. ${ }^{[23]}$ developed the heat transfer study by convection of a network based on the arboreal fractal branch. Through the model of convective heat transfer for laminar flow, it was concluded that the transfer rate increases with the increase of the pressure drop, the diameter of the branch and the difference in temperature; and on the other hand decreases with increasing the length of the structure.

The analysis of the receiver tube heat transfer on a parabolic cylindrical solar capture system based on a design under the branched fractal geometry is the objective of this work. In this work, the concentrator was designed by Euclidean parabolic geometry and the receiver tube by branched fractal geometry, seeking to improve the heat transfer phenomena such as radiation, convection, and conduction present in a CCP system.

\section{Methods}

To carry out the analysis developed in this work, a solar collector formed by a parabolic concentrator surface and a receiver designed under the fractal branch geometry for heat transfer was based on. Therefore, the description of branched fractal thermodynamics is described below.

The transfer of heat by radiation between two objects depends strongly on four factors; the distance of separation between them, the size, the relative form or orientation, and the material from which they are made. Each of these elements must be taken into account in the design stage of heat transfer systems. 


\subsection{Branched fractal geometry}

Considering that the fractal geometry is more successful in describing the natural forms and phenomena, than classical or Euclidean geometry, it was decided to use the fractal geometry as a basis of the design of a more efficient heat transfer system within a macro-solar collection system.

The branched fractal geometry, inspired by the natural structure of the trees, consists of the symmetrical branch in once iteration, where the dimensions will depend on the geometric scaling defined by the fractal dimensions. The parameters of the branched structure are length, diameter, and width for some cases, for these applications both the length and the diameter of the branch were considered. The structure based on the branched fractal geometry is shown in Figure 1.

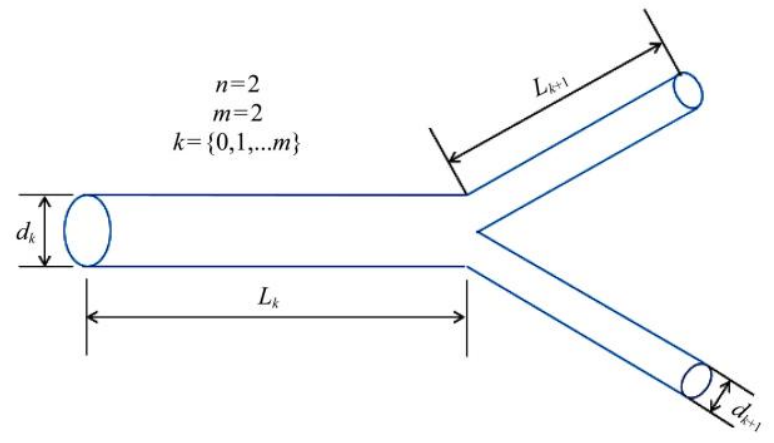

Figure 1 Branched fractal structure

Each branching level is defined by $k$, and the number of branches in once iteration is defined by $n$, so that the total number of branches $N$ in the entire network is obtained from Equation (1), for the total number of tree levels $m$.

$$
N=\sum_{k=0}^{m} N_{k}=\frac{\left(1-n^{m+1}\right)}{1-n}
$$

The scale factor for both length $Y_{k}(\mathrm{~m})$ and diameter $\beta_{k}(\mathrm{~m})$ is fixed for all iterations, so that the length $L_{k+1}(\mathrm{~m})$ and diameter $d_{k+1}$ (m) of the iteration depends on the values obtained by the last iteration, $L_{k}, d_{k}$ are calculated with Equation (2):

$$
Y_{k}=\frac{L_{k+1}}{L_{k}} ; \beta_{k}=\frac{d_{k+1}}{d_{k}}
$$

The scaling factors $Y_{k}$ and $\beta_{k}$ are related to the number of branches from the fractal dimension distributed both for length $D_{l}$, and for diameter $D_{d}$, as described in Equation (3):

$$
n=Y^{-D l}=\beta^{-D d}
$$

Once the scaling factors are defined, the total volume of the network $V\left(\mathrm{~m}^{3}\right)$ is obtained, from the number of total branches in each iteration and the own volume of the branches generated in that current iteration. Equation (4) expresses the volume of the network in terms of the number of branches, the diameter of the branch, and its length.

$$
V=\sum_{k=0}^{m} N_{k} V_{k}=\sum_{k=0}^{m} n^{k} \pi\left(\frac{d_{k}}{2}\right)^{2} L_{k}
$$

Similarly, from Equation (5), it is possible to obtain the volume of the network from the scaling factors and the parameters of the last branch of the tree.

$$
V=V_{m} n^{m}\left(\frac{1-\left(n Y \beta^{2}\right)^{-(m+1)}}{1-\left(n Y \beta^{2}\right)^{-1}}\right)
$$

where, $V_{m}$ is the volume of the terminal branch of the network. Another key parameter for the analysis in the area of the branch in each iteration $k\left(\mathrm{~m}^{2}\right)$, defined in Equation (6).

$$
A_{k}=\frac{\pi d_{k}^{2}}{4}
$$

By the law between electrical conductivity and network volume, the electrical resistance $R_{k}(\Omega)$ of a single tube is expressed in Equation (7), where $\rho$ is the resistivity of the material.

$$
R_{k}=\frac{\rho L_{k}}{A_{k}}=\frac{4 \rho L_{k}}{\pi d_{k}^{2}}
$$

The electrical resistance $R(\Omega)$ for the entire branched network can be defined as the sum of the resistance of each element (branch) of the iteration divided by the total number of branches for that same iteration, Equation (8).

$$
R=\sum_{k=0}^{m} \frac{R_{k}}{N_{k}}=\sum_{k=0}^{m} \frac{4 \rho L_{k}}{\pi n^{k} d_{k}^{2}}
$$

Once the electrical resistance of the network $R$ is obtained, it is possible to obtain the effective resistance $R_{e f}$ from the relationship shown in Equation (9).

$$
R_{e f}=\frac{R \times A_{e}}{L_{e}}
$$

where, $L_{e}$ is the equivalent length that depends on the length scaling and the value of the last branch, $\mathrm{m}$, as shown in Equation (10).

$$
L_{e}=\sum_{k=0}^{m} L_{k}=\frac{L_{m} \times\left[1-Y^{-(m+1)}\right]}{1-Y^{-1}}
$$

The value $A_{e}$, corresponds to the equivalent transversal area that relates the volume of the network and the equivalent length, as shown in Equation (11).

$$
A_{e}=\frac{V}{L_{e}}=\frac{\pi d_{k}^{2}}{4} \frac{n^{m}\left[\left(1-Y^{-1}\right)\left(1-\left(n Y \beta^{2}\right)^{-1}\right)\right]}{\left(1-Y^{-(m+1)}\right)\left(1-\left(n Y \beta^{2}\right)^{-(m+1)}\right)}
$$

For the inverse relationship between the effective resistivity of the network $R_{e f}$ and the effective electrical conductivity (EEC) $\sigma_{e}$ the latter is obtained as in Equation (12). The $\sigma$ value corresponds to the electrical conductivity of the material, $\mathrm{S} / \mathrm{m}$.

$$
\sigma_{e}=\frac{1}{R_{e f}}=\sigma\left[\frac{1-Y^{-(m+1)}}{1-Y^{-1}}\right]^{2}\left(\frac{1-\left(n Y \beta^{2}\right)^{-1}}{1-\left(n Y \beta^{2}\right)^{-(m+1)}}\right)\left(\frac{1-n \beta^{2} / Y}{1-\left(n \beta^{2} / Y\right)^{m+1}}\right)
$$

In cases where a fractal dimension of diameter equivalent to $D_{d}=2$ is defined and therefore the scaling factor is equal to $\beta=n^{-1 / 2}$, the effective electrical conductivity of the network reaches its maximum value in the electrical conductivity of the material defined for the fractal arrangement, this is due to the use of the function of volume and area preservation in the fractal filling system from one generation to another.

\subsection{Conduction heat transfer}

The total thermal resistance of the network $R_{e}\left(\mathrm{~W} / \mathrm{m}^{2} \cdot \mathrm{K}\right)$, solved by thermal-electric analogy is shown in Equation (13).

$$
R_{e}=\sum_{k=0}^{m} \frac{R_{k}}{N_{k}}=\frac{R_{m}}{n^{m}}\left[\frac{1-\left(n \beta^{2} / Y\right)^{m+1}}{1-n \beta^{2} / Y}\right]
$$

Assuming that the entire fractal network is part of a single tube the effective thermal conductivity (ETC) $\lambda_{e}$, is obtained in Equation (14), where $\lambda$ is the thermal conductivity of the material, $\mathrm{W} /(\mathrm{K} \cdot \mathrm{m})$.

$$
\lambda_{e}=\lambda\left[\frac{1-Y^{-(m+1)}}{1-Y^{-1}}\right]^{2}\left(\frac{1-\left(n Y \beta^{2}\right)^{-1}}{1-\left(n Y \beta^{2}\right)^{-(m+1)}}\right)\left(\frac{1-n \beta^{2} / Y}{1-\left(n \beta^{2} / Y\right)^{m+1}}\right)
$$

As EEC, the ETC reaches its maximum value equal to the thermal conductivity of the material, under the condition of preservation of area and volume. 


\subsection{Convection heat transfer}

For this analysis, it was considered that the Nusselt number remains constant for each branch level of the network. The system consists of a fully developed fluid with a constant heat flux in a uniform cross-section, which produces a temperature variation $\Delta T$, between the surface of the wall and the inner surface.

The convective heat transfer rate of the entire branched network $Q$ is the sum of each of the transfer rates in once iteration. In Equation (15), the convective heat is described.

$$
Q=\sum_{k=0}^{m} N_{k} h_{k} S_{k} \Delta T
$$

where, $h_{k}$ is the corresponding convective coefficient for each iteration, this will be incremented in each branch according to the scaling factor $\beta$, as illustrated in Equation (16).

$$
\frac{h_{k+1}}{h_{k}}=\frac{d_{k}}{d_{k+1}}=\frac{1}{\beta}
$$

From the relationships presented between the convective coefficient and the scaling in the diameter distribution, it is possible to define $h_{k}$ as in Equation (17).

$$
h_{k}=h_{k} \beta^{m-k}
$$

On the other hand $\left[S_{k}\right]$, corresponds to the area of heat transfer at the level $k$ of the branch, which can be defined as in Equation (18) for the proposed structure.

$$
S_{k}=\pi \cdot d_{k} \cdot L_{k}
$$

The total heat transfer area of the network $S$, it is possible to obtain from the sum of the areas $S_{k}$ described above and the branches number in each iteration $\left[N_{k}\right]$, as described in Equation (19).

$$
S=\sum_{k=0}^{m} N_{k} S_{k}=\sum_{k=0}^{m} n^{k} \pi d_{k} L_{k}
$$

Finally, the coefficient of effective convective heat transfer $h_{e f}$ in the entire network is related in Equation (20).

$$
h_{e f}=\frac{Q}{S \cdot \Delta T}
$$

The results obtained in this work are presented below, based on the analysis previously described in this section.

\section{Analysis of results}

For this analysis, a fixed number of branches was defined in each iteration $n=2$; for a fractal dimension in length $D_{l}=3$ and the fractal dimension in diameter $D_{d}=2$, this is because of the function of preserving volume and area in the fractal space-filling system generates a scaling factor $a=-\frac{1}{2}$.

\subsection{Modeling results}

The input parameters of the system are defined in Table 1. The material defined for this fractal structure is copper due to its high thermal properties.

Table 1 Fractal structure initial parameters

\begin{tabular}{cc}
\hline Parameter & Value \\
\hline$n$ & 2 \\
$D_{l}$ & 2 \\
$D_{d}$ & 2 \\
$Y$ & 0.7071 \\
$\beta$ & 0.7071 \\
$m$ & 4 \\
$L_{o} / \mathrm{m}$ & 0.0050 \\
$d_{o} / \mathrm{m}$ & 0.0050 \\
\hline
\end{tabular}

The branched fractal structure obtained with the parameters defined above is shown in Figure 2.

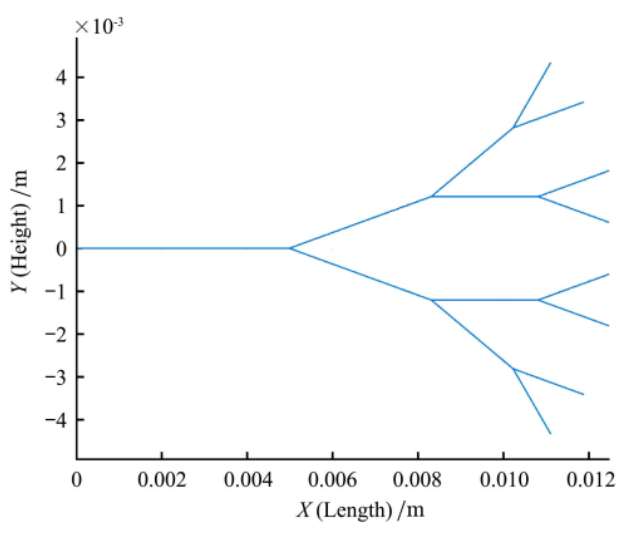

Figure 2 Branched fractal structure diagram

Due to the interest of this work of receiver tube heat transfer, the branched fractal structure obtained until the circular geometry of the tube was replicated, as illustrated in Figure 3.

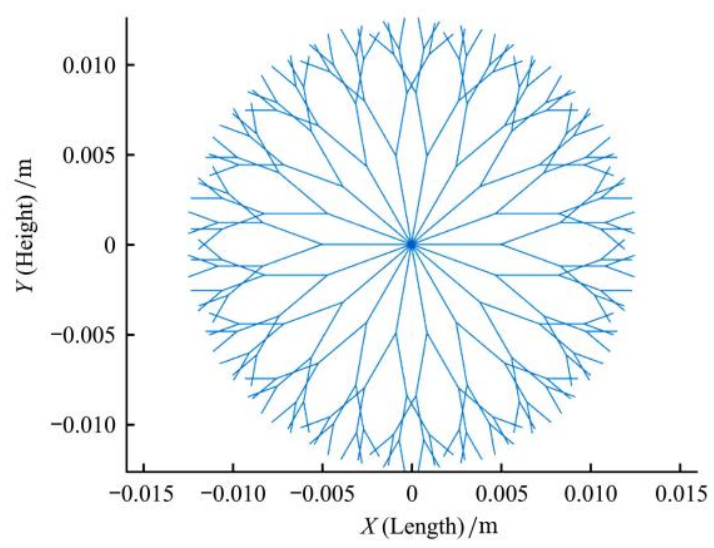

Figure 3 Branched fractal structure in the receiver tube

The results of the electric and thermal transfer according to the approaches described in the previous section are shown in Table 2.

Table 2 Thermal parameters obtained

\begin{tabular}{lc}
\hline \multicolumn{1}{c}{ Parameter } & Value \\
\hline$V / \mathrm{m}^{3}$ & $2.7594 \times 10^{-7}$ \\
$R / \Omega \cdot \mathrm{m}$ & $1.23105 \times 10^{-5}$ \\
$R_{e f} / \Omega \cdot \mathrm{m}^{2}$ & $1.72 \times 10^{-8}$ \\
$\sigma_{e} / \Omega^{-1} \cdot \mathrm{m}^{-1}$ & $5.81395 \times 107$ \\
$\lambda_{e} / \mathrm{W} \cdot \mathrm{m}^{-1} \cdot \mathrm{K}^{-1}$ & 372.1 \\
$S / \mathrm{m}^{2}$ & 0.000393 \\
$h_{e f} / \mathrm{W} \cdot \mathrm{m}^{-2} \cdot \mathrm{K}^{-1}$ & 11.8591 \\
\hline
\end{tabular}

The convective coefficients $h_{k}$ of each of the branches in the fractal structure are shown in Table 3.

Table 3 Network convection coefficients

\begin{tabular}{ccc}
\hline$k$ & $h_{k}$ & Coefficient $/ \mathrm{W} \cdot \mathrm{m}^{-2} \cdot \mathrm{K}^{-1}$ \\
\hline 0 & $h_{0}$ & 5.2742 \\
1 & $h_{1}$ & 7.4588 \\
2 & $h_{2}$ & 10.5483 \\
3 & $h_{3}$ & 14.9176 \\
4 & $h_{4}$ & 21.0966 \\
\hline
\end{tabular}

As expected, the convective coefficient increases in once iteration to the branches of higher level, in a scaling factor $\beta$. After obtaining the main structure together with its associated 
thermal, and electrical properties, an analysis was carried out where parameters such as the size of the fractal network $m$, the type of tube material and the fluid velocity $v_{f}$ were taken as independent variables. Through this, it was possible to demonstrate the effects on the thermal and electrical parameters of the network.

For a range of fractal size, comprised between 2 to 10 levels of branching, the structure obtained for a uniform tube with $m=10$ is illustrated in Figure 4.

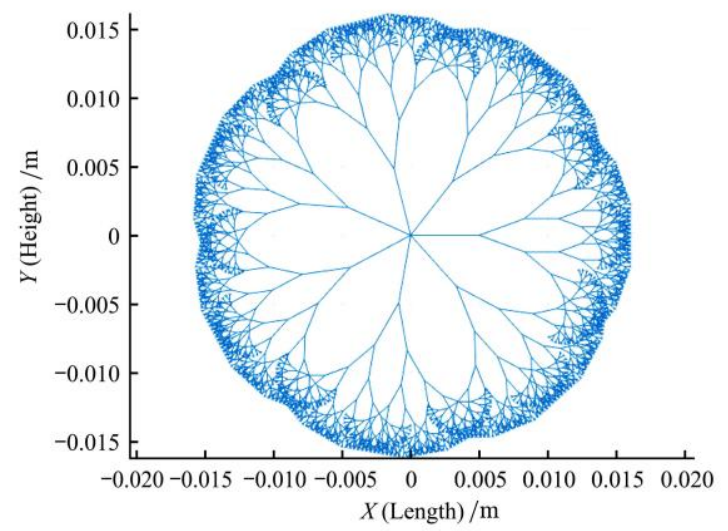

Figure 4 Network branched $m=10$

The electrical resistance of the entire network for each branching level is shown in Figure 5. Is evident that the network resistance behaves exponentially increasing as the branching level increases of the network; the maximum value reached for $m=10$ was $5.037 \times 10^{-5} \Omega \cdot \mathrm{m}$, increasing by $51.27 \%$ to resistance for $m=2$.

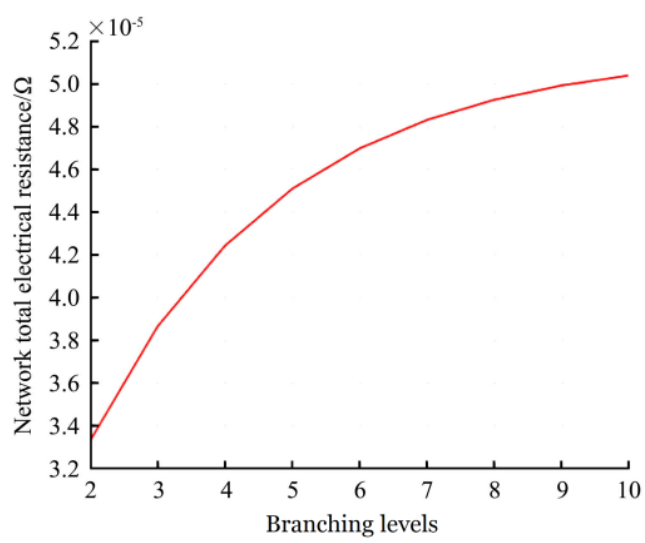

Figure 5 Electrical resistance to the variation of branching levels

Furthermore, the convective heat transfer presented a linear increasing behavior due to the variation of branching levels, as evidenced in Figure 6. The minimum value obtained for $m=2$, was $Q=899 \mathrm{~W}$ whereas the maximum value of heat transfer obtained was $Q=2.36 \times 10^{3} \mathrm{~W} \mathrm{~s}$, for $m=10$.

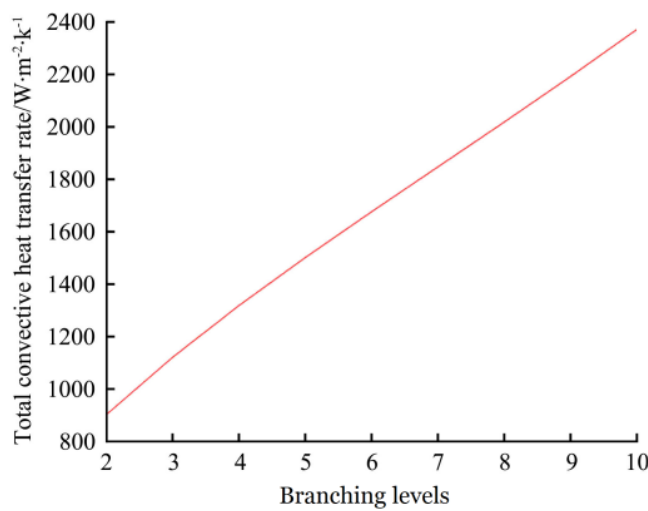

Figure 6 Convective heat transfer with respect to branching levels
Similarly, the area of heat transfer increased linearly as expected due to the variation of branches, the results are presented in Figure 7.

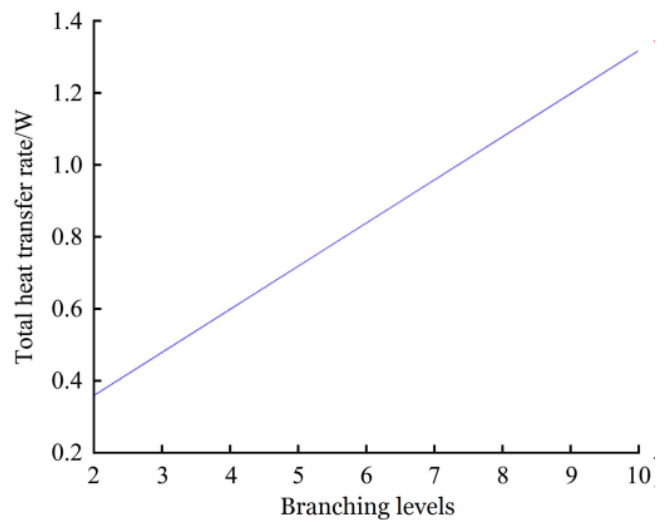

Figure 7 Heat transfer area with respect to branching levels

Finally, a decreasing exponential behavior of the effective convective coefficient before the variation of the ramifications was evidenced. An approximate variation of $28.17 \%$ was obtained between the maximum and minimum value of the coefficient. The graph of the behavior of this parameter is shown in Figure 8.

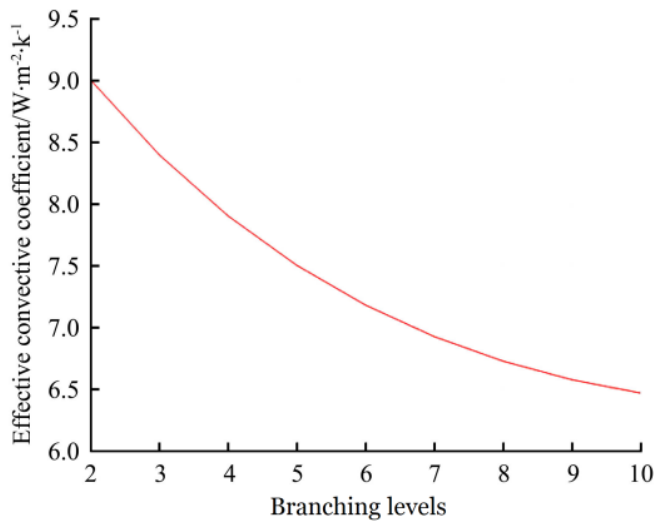

Figure 8 Effective convective coefficients with respect to branching levels

For different materials in terms of electrical resistivity, the analysis of the effective electrical resistance was carried out as shown in Figure 9. This parameter is highly increased for materials such as silver, steel, and iron; because they have greater resistance in their electrical properties than the other materials analyzed.

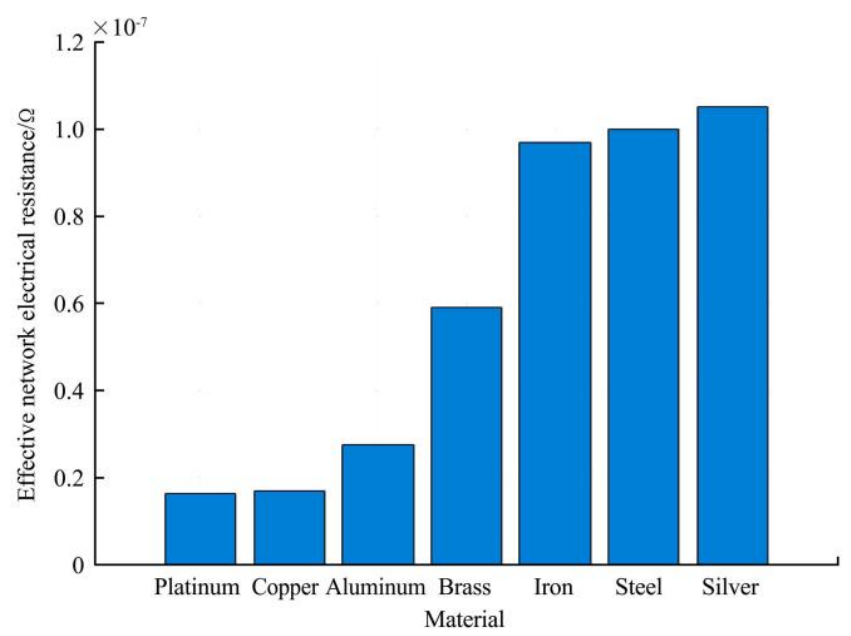

Figure 9 Effective electrical resistance of the network for different materials 
Similarly, it was possible to analyze the effective electrical conductivity of the network, where the highest conductivity was obtained for materials such as platinum and copper, while the lower conductivity was obtained in materials such as iron, steel, and silver Figure 10.

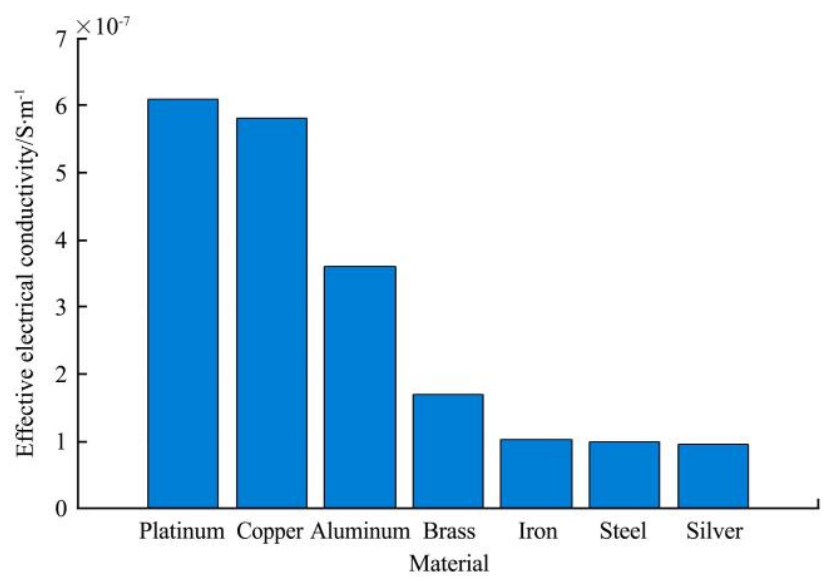

Figure 10 Electrical conductivity of the network for different materials

Finally, the effective thermal conductivity of the network, related in Figure 11, was obtained. From this, it is possible to visualize that the highest thermal conductivity is reached with silver, aluminum, and copper.

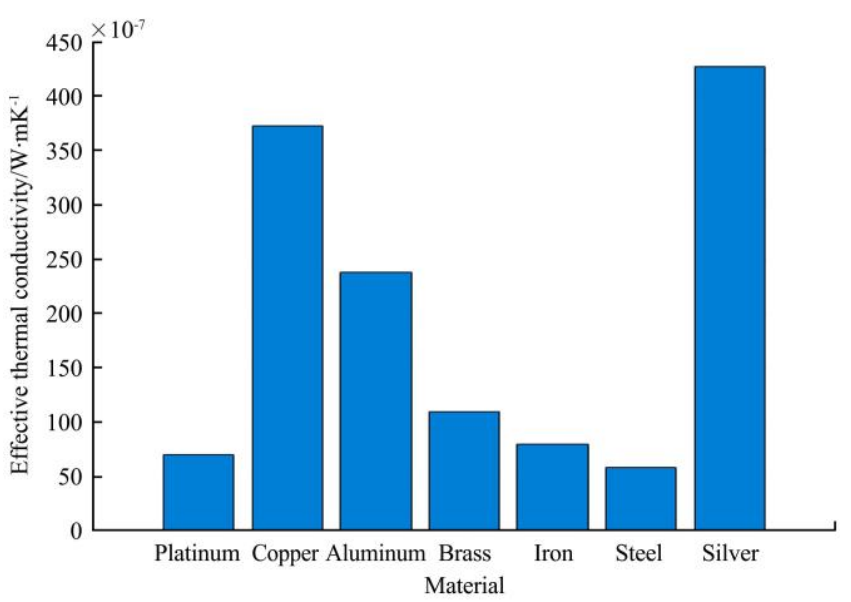

Figure 11 Network thermal conductivity for different materials

For a variable fluid velocity $1 \mathrm{~m} / \mathrm{s}$ and $3.5 \mathrm{~m} / \mathrm{s}$, the effective convective heat transfer coefficient obtained has a linear increasing behavior as opposed to the behavior obtained with the variation of the branching levels. The graph of Figure 12 shows what was described above.

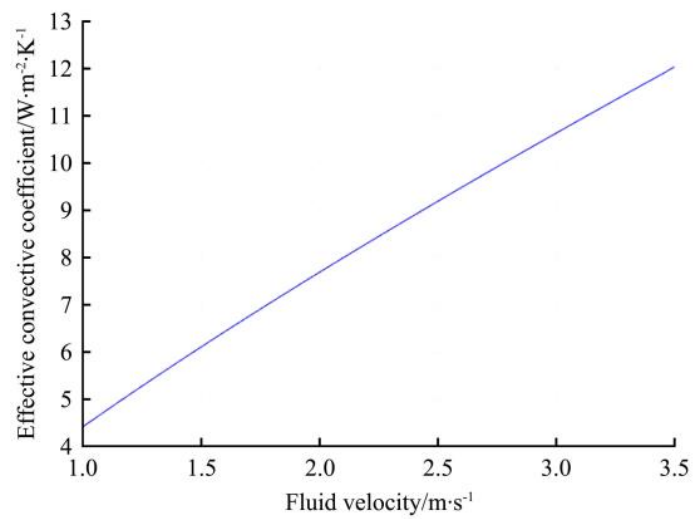

Figure 12 Effective convective coefficient with respect to $v_{f}$
Similarly, a behavior for convective heat was obtained by varying the velocity of fluid, the results are visualized in Figure 13.

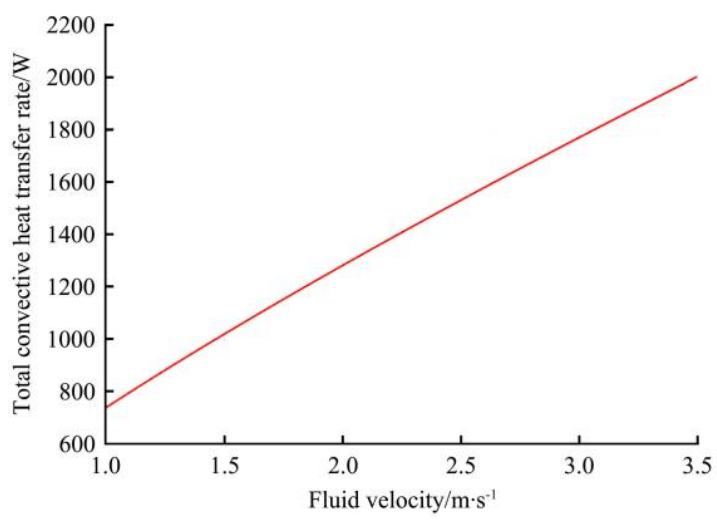

Figure 13 Convective heat before the variation of $v_{f}$

\subsection{CFD simulation results}

After analyzing the thermal transfer phenomena of the fractal branched network, we proceeded to define the physical parameters of the solar collector that would allow us to comply with the requirements of the receiver tube that contains the branched fractal geometry. The physical parameters of both the concentrator and the receiver are described in Table 4.

Table 4 Collector system parameters

\begin{tabular}{lcc}
\hline Parameter & Value & Description \\
\hline$L_{c} / \mathrm{m}$ & 3 & Concentrator length \\
$f / \mathrm{m}$ & 0.875 & Focal distance \\
$D_{r c} / \mathrm{mm}$ & 25.4 & Receiver diameter \\
$L_{r c} / \mathrm{m}$ & 3 & Receiver length \\
$D i_{r c} / \mathrm{mm}$ & 9.52 & Receiver internal diameter \\
$F_{c}$ & 43.8 & Concentration Factor \\
\hline
\end{tabular}

Considering the parameters presented above, the system was simulated through finite elements in the Solid Works program with the Flow Simulation tool. The branched network designed for the thermal analysis of this inside the receiver tube is shown in Figure 14 and Figure 15.

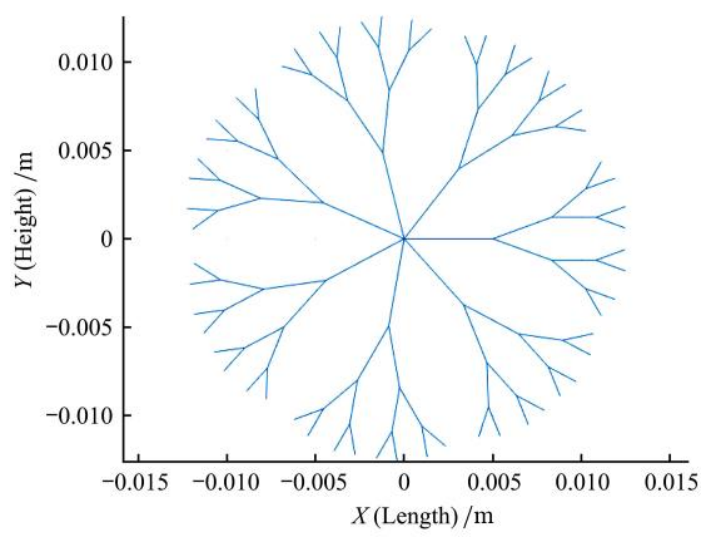

Figure 14 Branched network designed in Matlab

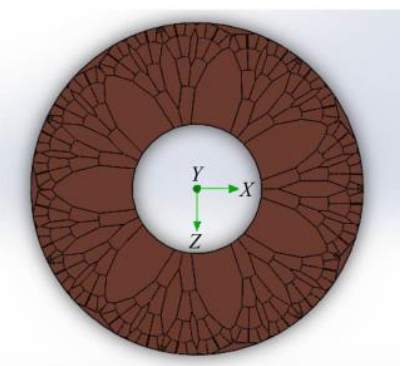

Figure 15 Branched network designed in Solid Works 
The interior of the receiver tube contains an internal tube where the different fractal branches are found, as illustrated in Figure 16.

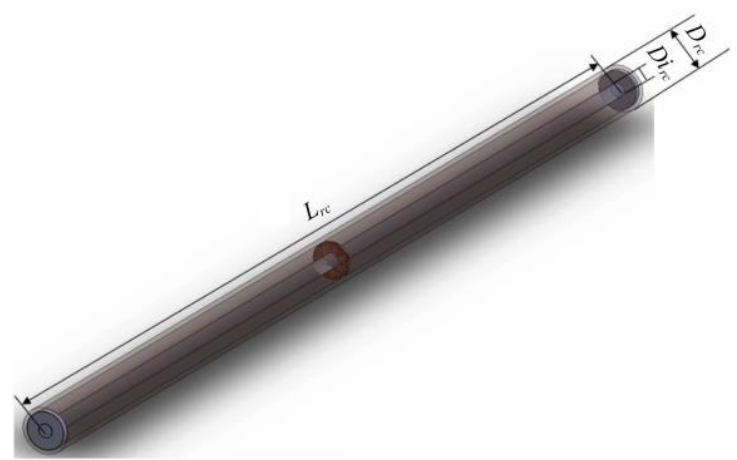

Figure 16 Cylindrical receiver

As shown in Figure 17, the fractal structure is located inside the cylindrical receiver, so it is in contact with both the internal and the external tube of the receiver.

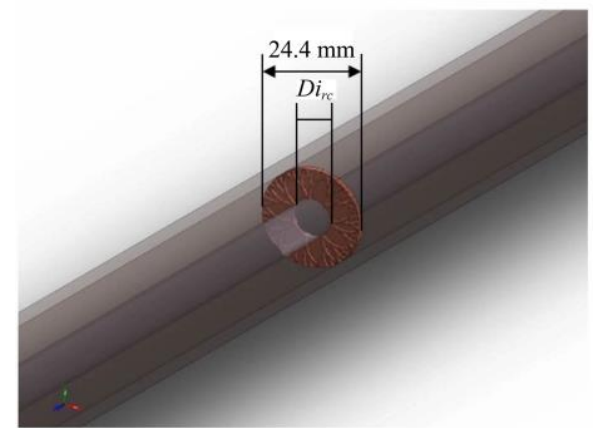

Figure 17 Fractal structure inside the receiver

The conformal CCP solar capture system by the branched receiver and the parabolic concentrator is presented in Figure 18.

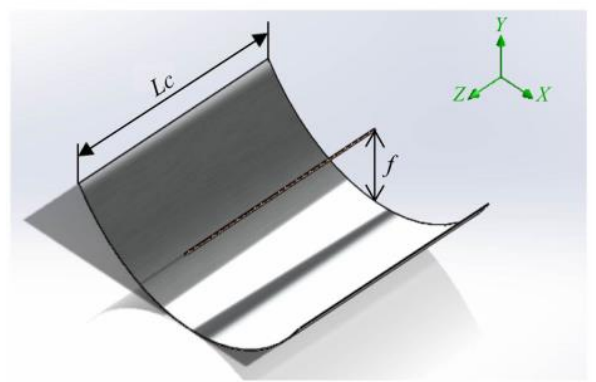

Figure 18 CCP system

The results obtained during the simulation are shown below in Table 5.
Table 5 Simulation results

\begin{tabular}{lcc}
\hline \multicolumn{1}{c}{ Parameter } & Minimum value & Maximum value \\
\hline Density (Fluid) $/ \mathrm{kg} \cdot \mathrm{m}^{-3}$ & 0.78 & 1.43 \\
Density (Solid)/kg $\cdot \mathrm{m}^{-3}$ & 8960.00 & 8960.00 \\
Pressure $/ \mathrm{Pa}$ & 101324.79 & 179179.58 \\
Temperature/K & 293.20 & 557.63 \\
Temperature (Fluid)/K & 293.20 & 557.59 \\
Temperature (Solid)/K & 388.81 & 557.63 \\
Velocity $/ \mathrm{m} \cdot \mathrm{s}^{-1}$ & 0 & 28.403 \\
Velocity $(X) / \mathrm{m} \cdot \mathrm{s}^{-1}$ & -0.055 & 0.055 \\
Velocity $(\mathrm{Y}) / \mathrm{m} \cdot \mathrm{s}^{-1}$ & -0.081 & 0.057 \\
Velocity $(\mathrm{Z}) / \mathrm{m} \cdot \mathrm{s}^{-1}$ & -28.403 & 0.035 \\
Mach number & 0 & 0.07 \\
Velocity RRF $/ \mathrm{m} \cdot \mathrm{s}^{-1}$ & 0 & 28.403 \\
Velocity RRF $(X) / \mathrm{m} \cdot \mathrm{s}^{-1}$ & -0.055 & 0.055 \\
Velocity RRF $(Y) / \mathrm{m} \cdot \mathrm{s}^{-1}$ & -0.081 & 0.057 \\
Velocity RRF $(\mathrm{Z}) / \mathrm{m} \cdot \mathrm{s}^{-1}$ & -28.403 & 0.035 \\
Vorticity $/ \mathrm{s}^{-1}$ & 0.02 & 7648.20 \\
Relative pressure/Pa & -0.21 & 77854.58 \\
Shear stress $/ \mathrm{Pa}$ & $5.64 \mathrm{e}-007$ & 2.36 \\
Heat flux $/ \mathrm{W} \cdot \mathrm{m}^{-2}$ & 2237.330 & 1593103.72 \\
Heat transfer coefficient $/ \mathrm{W} \cdot \mathrm{m}^{-2} \cdot \mathrm{K}^{-1}$ & $1.645 \mathrm{e}-005$ & 92.911 \\
Overheat above melting temperature $/ \mathrm{K}$ & -967.389 & -798.574 \\
Surface heat flux $/ \mathrm{W} \cdot \mathrm{m}^{-2}$ & -2259.588 & 22189.963 \\
Surface heat flux $(\mathrm{Convective}) / \mathrm{W} \cdot \mathrm{m}^{-2}$ & $-1.08 \mathrm{e}+007$ & 7080279.39 \\
\hline
\end{tabular}

The heat transfer inside the tube with the fractal structure is presented in Figure 19. Was possible to observe that the internal surface of the receiver, specifically in the fractal structure together with the internal walls, reaches the maximum temperature of $557626 \mathrm{~K}$.

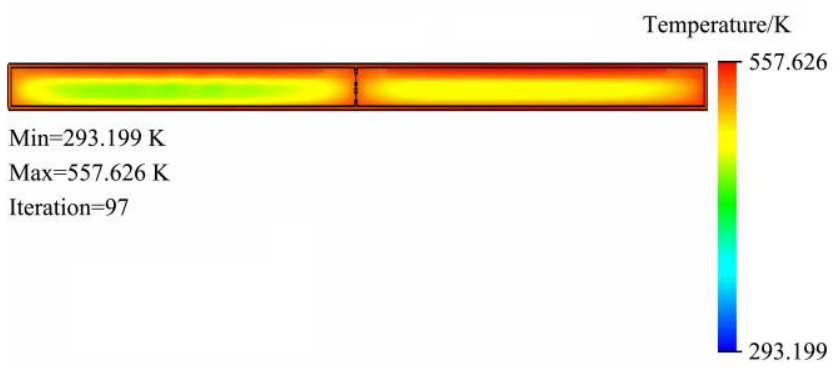

Figure 19 Internal temperature distribution

The airflow and its temperature inside the internal receiver are shown next to the temperature of the surface of the branched fractal structure in Figure 20.

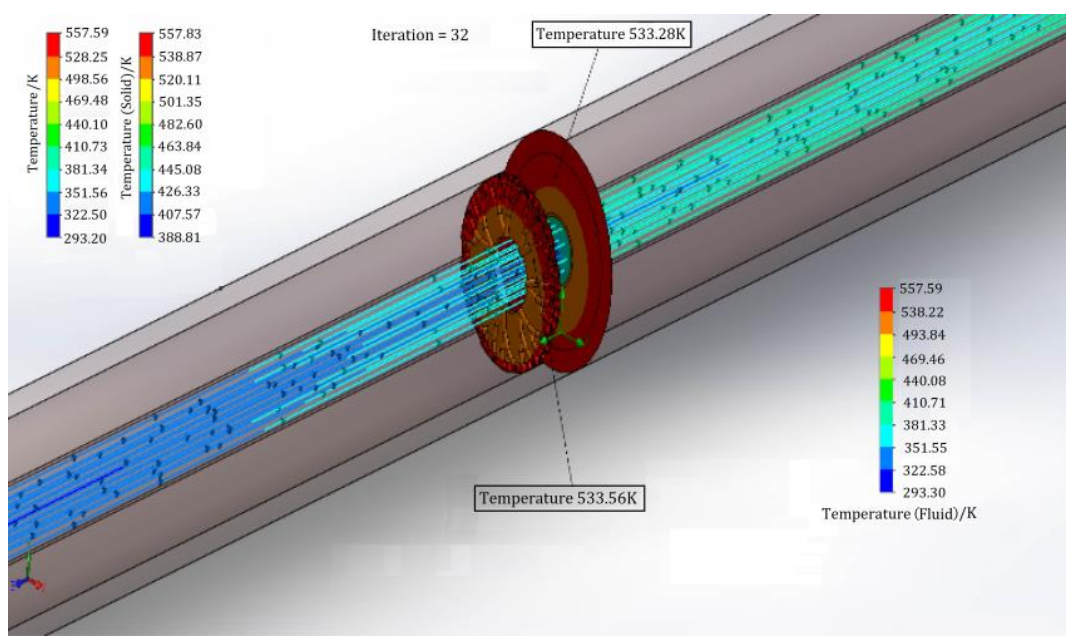

Figure 20 Inside fluid trajectory of the receiver 
The temperature distribution on the surface of the branched fractal structure is visualized in Figure 21. The heat is transferred from the inside to the outside of the network, in higher-level branches higher temperature is reached, the temperature delta between the inside and outside of the fractal network is $20.15 \mathrm{~K}$.

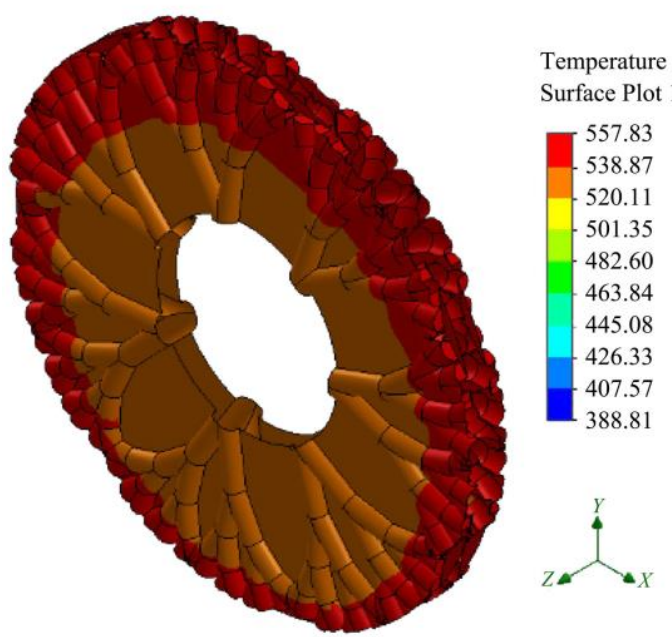

Figure 21 Temperature distribution in the fractal structure

\section{Conclusions}

The branching levels number of an arboreal fractal type network highly influences parameters such as electrical resistance, thermal resistance, and convective heat transfer; which grow exponentially or linearly to a greater number of branches in the Network; unlike these, the convective coefficient decreases exponentially with the increase in the size of the fractal structure.

Depending on the material, the electrical and thermal resistance is superior for materials such as iron, steel and silver; while the electrical conductivity is low for these and high for platinum, copper and aluminum. Due to the above and at costs, the material selected for the fractal structure of the receiver tube was copper, with which maximum temperatures of $557626 \mathrm{~K}$ were obtained in the upper levels of the network and an approximate temperature of $535 \mathrm{~K}$ in the lower levels.

The heat transfer from the fractal structure to the receiver tube was evidenced by finite element simulation, with which the heat distributions from the inside to the outside of the tube showed a temperature delta of $20.50 \mathrm{~K}$; while the temperature delta between the solid and the fluid reached was $0.04 \mathrm{~K}$; with a maximum fluid temperature of $557.59 \mathrm{~K}$; therefore branched fractal structures improve heat transfer between conductive surfaces.

\section{Acknowledgements}

The authors acknowledge that this work was financially supported by the Universidad Militar Nueva Granada, Research Vice-rectory and research center (IMP-ING-2656, 2018).

\section{[References]}

[1] Nesshöver C, Assmuth T, Irvine K N, Rusch G M, Waylen K A, Delbaere $\mathrm{B}$, et al. The science, policy and practice of nature-based solutions: An interdisciplinary perspective. Science of the Total Environment, 2017; 579: 1215-1227.

[2] Huang Z W, Hwang Y H, Aute V, Radermacher R. Review of Fractal Heat Exchangers. Int. Refrig. Air Cond. Conf., 2016; 1725, 9p.

[3] Lee J, Yi J Y, Kim L H, Shin C B. Dynamic modeling of the hydraulic-thermal behavior of the buried pipe network for district heating. J. Energy Eng., 2012; 21(2): 144-151.

[4] Cai J, Perfect E, Cheng C L, Hu X. Generalized modeling of spontaneous imbibition based on hagen-poiseuille flow in tortuous capillaries with variably shaped apertures. Langmuir, 2014; 30(18): 5142-5151.

[5] Wang X Q, Xu P, Mujumdar A S, Yap C. Flow and thermal characteristics of offset branching network. Int. J. Therm. Sci., 2009; 49: 272-280.

[6] Emerson D R, Cieślicki K, Gu X, Barber R W. Biomimetic design of microfluidic manifolds based on a generalised Murray's law. Lab Chip, 2006; 6(3): 447-454.

[7] Falconer K. Fractal geometry: Mathematical foundations and applications. John Wiley \& Sons, 2003; 155p

[8] Liu D J, Zhou W G, Wu J, Huang T F. Fractal characterization of graphene oxide nanosheet. Materials Letters, 2018; 220: 40-43.

[9] Gil J C A, Cherkashinin Y G, Korili S A. Fractal dimension of a pillared montmorillonite from nitrogen adsorption at 77 K. J. Chem. Eng., 2004; 49(3): 639-641.

[10] Klioutchnikov I, Sigova M, Beizerov N. Chaos Theory in Finance. Procedia Comput. Sci., 2017; 119: 368-375.

[11] Chellam S, Wiesner M R. Fluid mechanics and fractal aggregates. Water Res., 1993; 27(9): 1493-1496.

[12] Zuo R, Wang J. ArcFractal: An ArcGIS add-in for processing geoscience data using fractal/multifractal models. Nat. Resour. Res., 2020; 29(1): $3-12$.

[13] Hossain M S. Fractals: Concepts and applications in geosciences Annual General Meeting of the Geological Society of India, 2016; 234p.

[14] Balankin A S. Mapping physical problems on fractals onto boundary value problems within continuum framework. Phys. Lett. A, 2018; 382(4): 141-146.

[15] Vittitoe N F, Baker J A, Floyd C E. Fractal texture analysis in computer-aided diagnosis of solitary pulmonary nodules. Acad. Radiol., 1997; 4(2): 96-101.

[16] Tarasov V E. Heat transfer in fractal materials. Int. J. Heat Mass Transf., 2016; 93: 427-430.

[17] Nayak S R, Mishra J, Khandual A, Palai G. Fractal dimension of RGB color images. Optik (Stuttg), 2018; 162: 196-205.

[18] Tang Z, Wu X, Fu B, Chen W, Feng H. Fast face recognition based on fractal theory. Appl. Math. Comput., 2018; 321: 721-730.

[19] Ma Y J, Zhai M Y. Fractal and multi-fractal features of the broadband power line communication signals. Comput. Electr. Eng., 2018; 72: $566-576$.

[20] Veitia-Rodríguez D E R, Villegas-Zulueta A, Martínez-López Y, Montalbán-Estrada A. Fractal dimension of global change and environmental sustainability soil. Hombre, Cienc. y Tecnol., 2013; 17(1): 73-82.

[21] Xu P, Yu B. The scaling laws of transport properties for fractal-like tree networks. J. Appl. Phys., 2006; 100(10): 207. doi: 10.1063/1.2392935.

[22] Xu P, Yu B, Yun M, Zou M. Heat conduction in fractal tree-like branched networks. Int. J. Heat Mass Transf., 2006; 49(19-20): 3746-3751.

[23] Luo L, Tian F, Cai J, Hu X. The convective heat transfer of branched structure. Int. J. Heat Mass Transf., 2018; 116: 813-816. 